ORIGINAL ARTICLE

\title{
Toxic Effects of Different Doses of Cyclophosphamide on Liver and Kidney Tissue in Swiss Albino Mice: A Histopathological Study
}

\author{
Nandini Bhat ${ }^{1}$, Sneha Guruprasad Kalthur ${ }^{*}$, Supriya Padmashali ${ }^{2}$, Vidya \\ Monappa $^{3}$
}

\footnotetext{
OPEN ACCESS

Citation: Nandini Bhat, Sneha Guruprasad Kalthur, SupriyaPadmashali, VidyaMonappa. Toxic Effects of Different Doses of Cyclophosphamide on Liver and Kidney Tissue in Swiss Albino Mice: A Histopathological Study. Ethiop J Sci.2018;28(6):711.

ddoi:http://dx.doi.org/10.4314/ejhs.v28i6.5

Received: February 5, 2018

Accepted: April 19, 2018

Published: November 1, 2018

Copyright: (C) 2018 nandini B., et al . This is an open access article distributed under the terms of the Creative Commons Attribution License, which permits unrestricted use, distribution, and reproduction in any medium, provided the original author and source are credited. Funding: Cost of sample transporting was supported by Bule Hora University.

Competing Interests: The authors declare that this manuscript was approved by all authors in its form and that no competing interest exists.

Affiliation and Correspondence:

${ }^{1}$ Department Of Anatomy, Kasturba

Medical College, Basic Sciences

Building, Manipal Academy of Higher

Education, Manipal 576104,

Karnataka, India

${ }^{2}$ Department of Anatomy, Kannur

Medical College, Kannur, Kerala

${ }^{3}$ Department of Pathology, Kasturba

Medical College, Basic Sciences

Building, Manipal Academy of Higher

Education, Manipal 576104,

Karnataka, India

*Email:sneha.guruprasad@manipal.edu
}

\begin{abstract}
BACKGROUND: Cyclophosphamide (CPA) is an anti- cancer drug, used in chemotherapy. This is a toxic drug which targets the cancer cells and also the normal cells of the body. The original compound is inactive in vitro and exercises its biologic action through metabolites, chiefly phosphoramide mustard. The objective is to study the harmful effects of this drug on liver and kidney tissues.

METHODS: To study the effect of cyclophosphamide on histology of liver and kidney, 40 adult male mice were taken and divided into two groups: control and test. Those in the test group were injected with the drug at doses of 100, 200, $250 \mathrm{mg} / \mathrm{kg}$ body weight. They were then sacrificed on day 7, 28 and 42. The liver and kidney tissue was processed, sectioned and stained with Haematoxylin and Eosin.

RESULTS: Pathological changes were seen in the tissue within 7 days in high doses and after 28 days in low doses. As the dosage and the days administered increased, the changes were prominently seen and widespread. Pathology ranging from mild infiltration to necrosis and finally cytolysis were seen in liver and kidney tissue.

CONCLUSION: Our study has demonstrated the effect of a progressive increase in dosage of cyclophosphamide in albino mice, and pathological alterations were observed in histology of liver and kidney by sequentially increasing both the dosage and duration of treatment. Subsequently, regular monitoring of liver and kidney function tests in patients undergoing chemotherapeutic regimen with administration of ahepato and nephroprotective agent becomes vital.
\end{abstract}

KEYWORDS: cyclophosphamide, histopathology, hepatotoxicity, nephrotoxicity, chemotherapy

\section{INTRODUCTION}

Cyclophosphamide (CPA) is an oxazaphosphorine class of alkylating agent. An alkylating agent is a class of drugs that bind to 
anucleophilic substance of DNA of the cell and prevent

original compound is inactive in vitro and exercises its biologic action through metabolites, chiefly phosphoramide mustardwhich is produced by hepatic microsomal enzymes P450 system. However more than 150 metabolites have been documented so far, but their pharmacokinetics and harmfulness are not well demarcated (2). The main use of CPA is as a chemotherapy drug permitted for usage in the United States in 1959, and its existing indications include management of breast cancer, nonHodgkin's lymphoma, acute myeloid leukemia, chronic myelogenous leukemia and acute lymphoblastic leukemia (3). CPA treatment for 3 days showed bone marrow suppression, significantly decreasing hemoglobin, $\mathrm{RBC}$, and $\mathrm{WBC}$ levels. It also acts on the defense mechanism inhibiting both cell-mediated and humoral immunity (4).

As it also exhibits immunosuppressive actions, it is frequently used in autoimmune disorders such as Nephrotic syndrome, and organ transplant treatment regimens to prevent rejection. CPA prevents cellular/ humoral rejection and maintains immunosuppression without requiring the supplementation of corticosteroids (5).The recommended dosage of CPA for treatment differs with the duration of the disease, the weight of the patient and the route of administration (6).

Although it is very active in killing tumor cells and plays a key role in immunosuppression, it also displays a wide range of toxic effects. Oral administration of $10 \mathrm{mg} / \mathrm{kg}$ of CPA for 30 days induced decrease in body weight and delayed hypersensitivity reaction in Wisteria rats. The histopathology of spleen and thymus showed asignificant decrease in cellularity. The CPAinduced liver injury is mainly dose-dependent. Apparently, it is a consequence of compromised metabolism of CPA, particularly of its metabolite, acrolein (6).

Increased exposure to metabolites of $\mathrm{CPA}$ leads to hepatotoxicity, symptoms of liver dysfunction related to sinusoidal obstruction syndrome with raised level of aminotransferase and bilirubin (7). In themajority of cases with cancer, which were treated with cyclophosphamide, slight and transient elevations in serum aminotransferase levels were observed.The rise in the level of enzymes is more seen in cases treated with high doses of CPA administered directly into the blood through intravenous route (8).

Regular doses of cyclophosphamide producing clinically apparent liver and kidney injuries are unusual. The commencement is within the first few weeks of CPA therapy, and initially increase in serum enzymes is observed. The destruction in most cases is self-limited, and resolutions areapparent on discontinuing the drug with a recurrence on reexposure (9).

In the literature, several experimental animal studies with CPA have been done. It shows the adverse effects of CPA on the tissues. A study on histopathology of the liver of Swiss albino mice shows signs of hepatic degeneration (10-11). Previous studies focussed mainly on a few optimal concentration that could be toxic to the cells. However, in the present study different doses in increasing order were administered to the animals. Additionally,tissues were procured at regular intervals, unlike former studies which processed tissues at the end of the treatment plan. The aim of this study was to examine the histology of liver and kidney of Swiss albino mice acquired at regular intervals after treatment with CPA in increasing doses.

\section{MATERIALS AND METHODS}

Animal model: Healthy male Swiss albino (Mus musculus) mice from inbred colony maintained at Central Animal Research Facility (Reg No 94/1999/CPCSEA) were used for the study. Forty adult mice with average weight of $32 \pm 5 \mathrm{~g}$ were maintained in well ventilated polypropylene cages containing paddy husk. Animals were given food and fresh water ad libitum. The protocol for animal use was approved from the Institutional Animal Ethics Committee (IAEC Ref No-79/2013).

Experimental design: The adult male mice were divided into two groups; control $(\mathrm{n}=6)$ and test $(n=34)$. Since the daily oral therapeutic dose of cyclophosphamide for human beings is $5 \mathrm{mg} / \mathrm{kg}$ body weight so from this dose the dose for albino rats was calculated $(3,12)$. In the test group, the mice were intra-peritoneally injected with CPA single dose of 100,200 and $250 \mathrm{mg} / \mathrm{kg}$ body weight. From each group, 4 mice were sacrificed on days 7, 28

DOI: http://dx.doi.org/10.4314/ejhs.v28i6.5 
and 42. The control mice were injected with Phosphate Buffered Saline (PBS) solution.

Collection of liver and kidney: On the specific period, animals were sacrificed by cervical dislocation. The mice were dissected as per guidelines given in "The Laboratory MouseHandbook of experimental animals" by Hans Hedrich. A midline incision was given on the abdomen with the help of scalpel, the organs liver and kidney were identified and dissected out. The tissues were fixed in modified Bouin's solution $(0.2 \%$ picric acid $2 \%(\mathrm{v} / \mathrm{v})$ formaldehyde in PBS) and then transferred to $70 \%$ alcohol for histological examination.

Histological technique: The tissues stored were processed by dehydration in $90 \%$ alcohol, absolute alcohol and finally dipped in xylol. The organs (liver and kidney) were embedded in paraffin wax and blocks were prepared and labelled. $5 \mu \mathrm{m}$ thickness sections were cut using rotatory microtome. The sections were fixed on slides and stained using Haematoxylin and Eosin according to the guidelines given in the manual Principles and Techniques in Histology Microscopy and Photomicrography by D. R. Singh (13).

Histological assessment: Stained sections were studied under Olympus Research Microscope (model cx21i) and photographed at $10 \mathrm{X}$ and $100 \mathrm{X}$ magnification in at least three random microscopic fields from each animal by expert pathologist without knowledge of the treatment groups. Three factors such as hepatocellular necrosis, level of inflammatory in-portal area, and lymphocytic inflammatory infiltrations were evaluated using semiquantitative method. Similarly, from kidney sections, the structures of cortex, medulla and tubules were scrutinized.

\section{RESULTS}

Control group: Histological examination of liver tissue in this group displayed normal microstructure. Distinct hepatic lobules were seen, cords of hepatocytes were separated with sinusoids. Central vein and portal triad were also perceived to be normal (Figure 1.1).

100mg CPA-treated group: At 7 days, mild histological changes were seen in this group. In animals sacrificed at 7 days, the changes in liver seen were mild peri-venular infiltration. The hepatocytes and sinusoids appeared normal (Figure 1.2). In kidney sections, some areas of cortex showed infiltrate. There was rarefaction of cytoplasm of the tubules in the junction of cortex and medulla. (Figure 4.2).

At 28 days: The central vein and portal veins of liver were engorged. The hepatocytes still appeared normal. Multinucleated megakaryocytes were seen in the hepatic lobules (Figure 1.3).

Kidney: Inflammatory cells seen are same as previous sections. Outer most cortex shows swelling of cells and rarefaction of cytoplasm which was present in the junction in the previous sections. Medulla also shows swollen cells (Figure 4.3).

At 42 days: Slight enlargement of hepatocytes in liver were seen; there was increased infiltration of inflammatory cells into the liver tissue. There is both central and portal venous engorgement (Figure 1.4)

Kidney: A large number of pyknotic nucleus was seen in the cortex. Inflammatory cells increased in the outer cortex. (Figure 4.4)

200mg CPA-treated group: In this group, the changes were seen earlier than the previous group.

At 7 days on liver: The hepatocytes and sinusoids showed no change from the control group. Megakaryocytes were seen in between the tissue (Figure 2.2).

Kidney: Inflammatory cells reduced. Oedematous changes of the tubules in cortex is more striking. Cortex shows sloughing off cells. Pyknotic nucleus present in thecortex. Medulla shows only swelling of cells and no other major changes (Figure 5.2).

At 28 days: Hepatocytes were swollen, sinusoids were congested. Portal and central vein were normal and the infiltrate absent. (Figure 2.3).

Kidney: Pyknotic nucleus seen in cortex, loss of brush border of the tubules. Congestion of the vessels is reduced. Inflammatory cells reduced. Medulla shows swollen cells. (Figure 5.3).

At 42 days: The hepatocytes were enlargedballooning effect. Sinusoids were markedly congested and periductular necrosis is present. (Figure 2.4).

Kidney: Swollen cells of PCT with theoccasional absence of anucleus. Inflammatory cells increased. Congestion of blood vessels is minimal. Medulla shows large cells. (Figure 5.4).

$250 \mathrm{mg}$ CPA-treated group: As this is ahigh dose, the toxic effects were markedly seen on the

DOI: http://dx.doi.org/10.4314/ejhs.v28i6.5 
microstructure of liver tissue. The changes were more pronounced.

At 7 days: The hepatocytes were swollen, sinusoids were enlarged, perivenular spotty necrosis and venous engorgement was seen (Figure 3.2).

Kidney: No inflammatory cells seen. Increased number of pyknoticnuclei. Blood vessels show marked congestion. Medulla shows increase in swelling of cells. (Figure 6.2).

At 28 days on liver: The normal structure of hepatic lobule was lost. There was cytolysis that is the cell membrane wasdamaged;Nucleuswas present whichwas small and pyknotic. There was minimal infiltration but marked dilatation of veins (Figure 3.3).
Kidney: congestion of blood vessels present. No presence of inflammatory cells. Normal nucleus. Swelling of cells all throughout medulla and cortex. (Figure 6.3).

At 42 days on liver: Cytolysis with pyknotic nucleus was seen. Spotty necrosis and marked venous engorgement of veins. Normal architecture of liver tissue was damaged. The cords of hepatocytes were no longer seen. The sinusoids were disrupted, the veins were significantly dilated occupying large areas. (Figure 3.4).

Kidney: Appearance of pyknotic nucleus, oedema of cells and loss of brush border same as thelast section. (Figure 6.4).
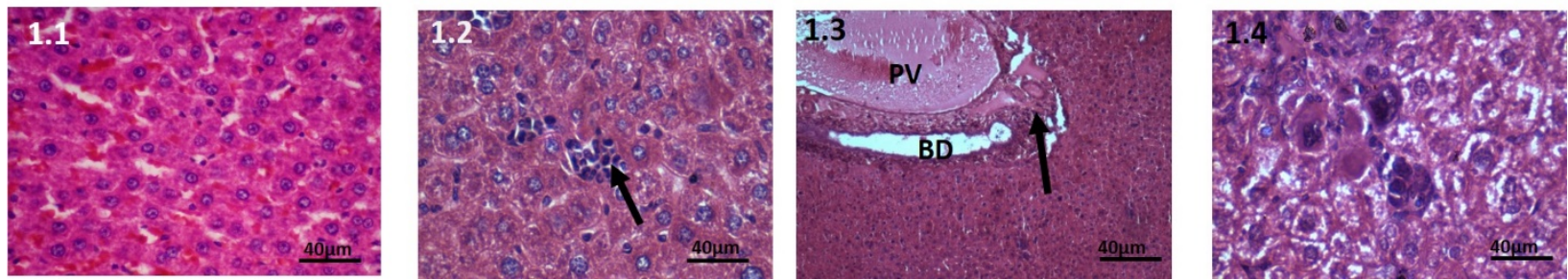

Figure 1: Haematoxylin and Eosin stained liver tissue, 40X, 100mg CPA treated group. 1.1: Control, Liver tissue shows normal hepatocytes and sinusoids. 1.2: At 7 days: Multinucleated megakaryocytes are seen. 1.3: At 28 days, The portal vein is engorged. Mild periductular infiltration. 1.4: At 42 days, Slight enlargement of hepatocytes are seen, there is increased infiltration of inflammatory cells.
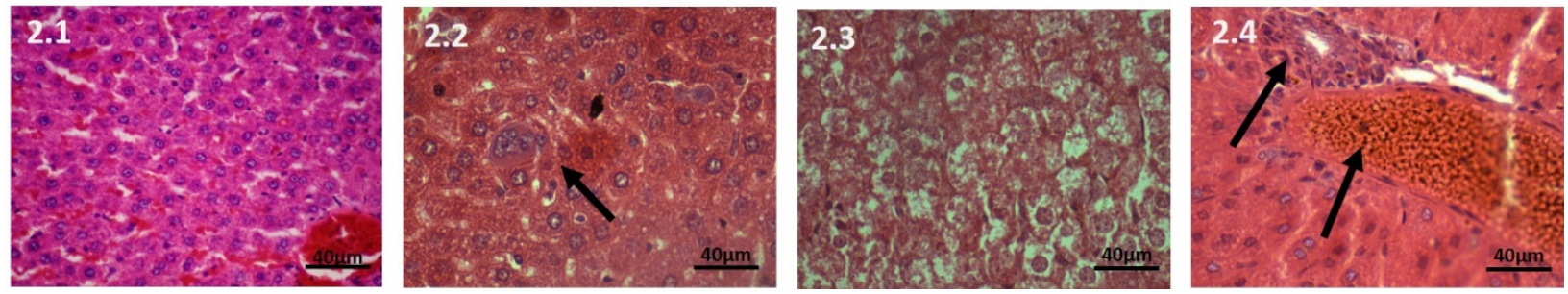

Figure 2: Haematoxylin and Eosin stained liver tissue, 40X, 200mg CPA treated group. 2.1: Control, The hepatocytes and sinusoids are normal. 2.2: At 7 days, The hepatocytes and sinusoids are normal. Megakaryocytes are seen in lobules. 2.3: At 28 days, Hepatocytes are swollen, sinusoids are congested. 2.4: At 42 days, The hepatocytes are enlarged- ballooning effect. Enlarged portal vein and periductular necrosis is present
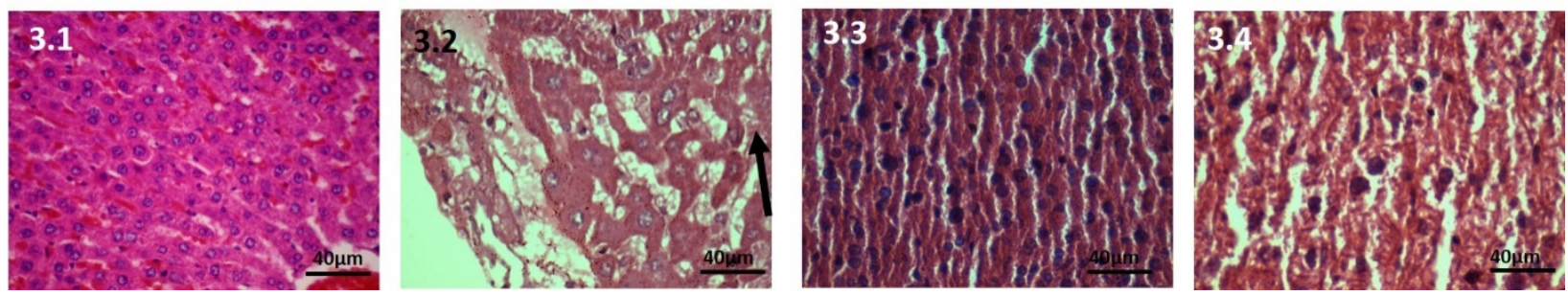

Figure 3: Haematoxylin and Eosin stained liver tissue, 40X, 250mg CPA treated group. 3.1: Control, The hepatocytes, sinusoids and veins normal. 3.2: At 7 days, The hepatocytes are swollen, sinusoids are enlarged. 3.3: At 28 days, The normal structure of hepatic lobule is lost. There is cytolysis that is the cell membrane is damaged, Nucleus is present which is small and pykonotic. There is minimal infiltration. 3.4: At 42 days, Cytolysis with pykonotic nucleus is seen. Spotty necrosis and marked venous engorgement of veins. Normal architecture of liver tissue is damaged. The cords of hepatocytes are no longer seen. The sinusoids are disrupted and are significantly dilated occupying large areas.

DOI: http://dx.doi.org/10.4314/ejhs.v28i6.5 

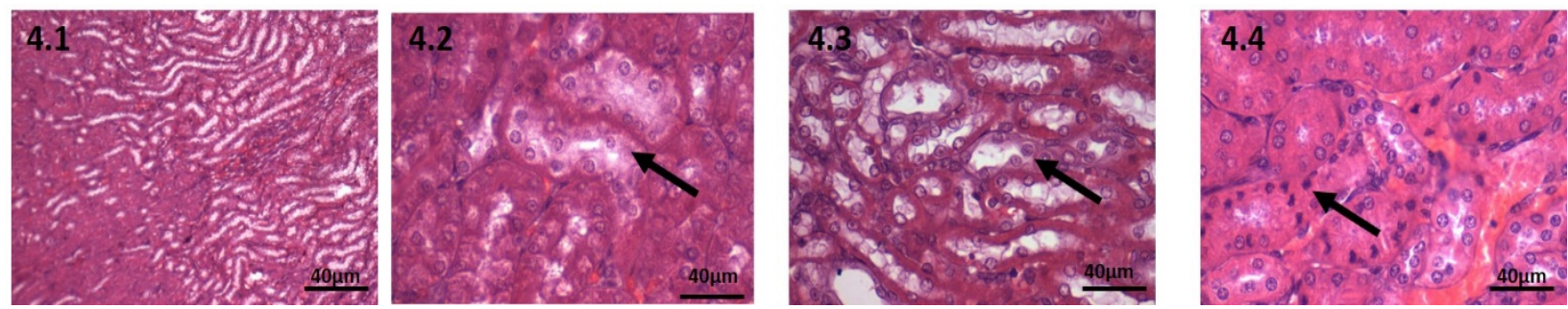

Figure 4: Haematoxylin and Eosin stained kidney tissue, 40X, 100mg CPA treated group. 4.1: Control, Kidney cortex and medulla shows normal architecture 4.2: At 7 days, rarefaction of cytoplasm of the tubules in the cortex. 4.3: At 28 days, Swollen cells of tubules, 4.4: At 42 days, pyknotic nucleus seen both in cortex and medulla
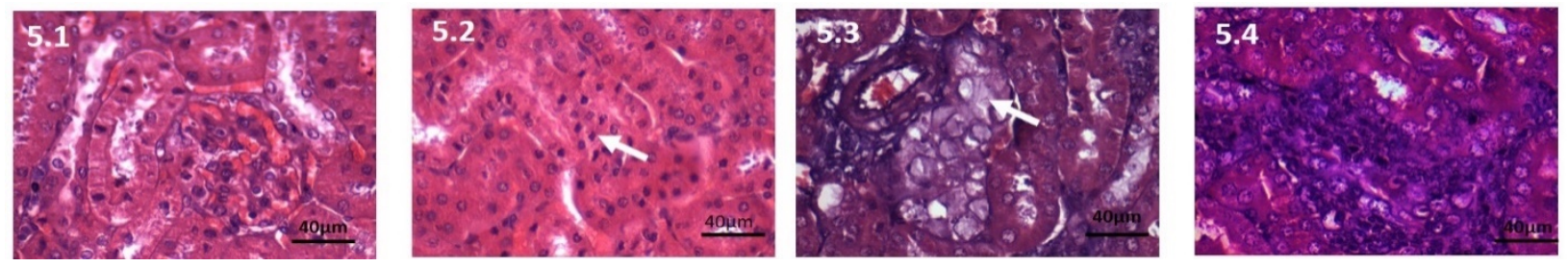

Figure 5: Haematoxylin and Eosin stained kidney tissue, 40X, 200mg CPA treated group 5.1: Control, Kidney section showing normal structure. 5.2: At 7days, Pyknotic nuclei increased. 5.3: At 28 days, swollen cells occasional nucleus absent. 5.4: At 42 days, No inflammatory cells, pykontic nucleus.
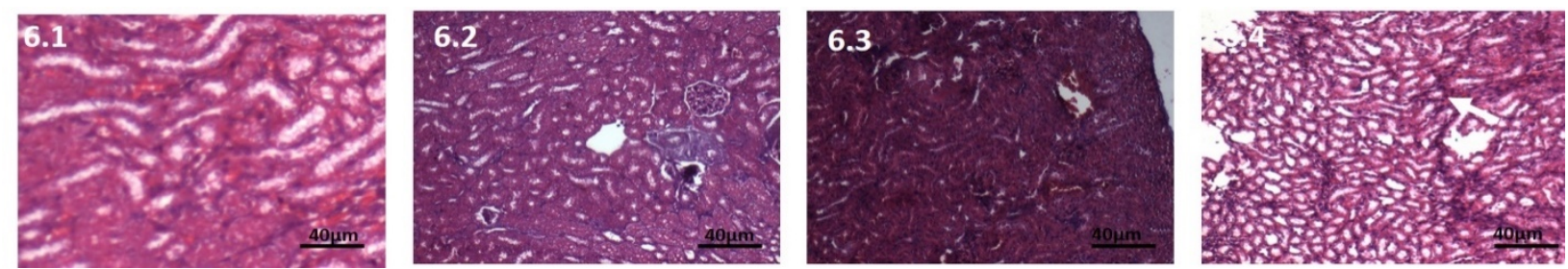

Figure 6: Haematoxylin and Eosin stained kidney tissue, 10X, 250mg CPA treated group 6.1: Control, Kidney section showing normal structure under higher magnification; 6.2: At 7 days, Congestion, swelling of tubules, loss of brush border; Fig 6.3: At 28 days, Marked congestion; 6.4: At 42 days, Necrotic tissue seen

\section{DISCUSSION}

Cancer in the human body is treated with numerous diverse chemotherapeutic agents. The therapeutic drugs can be categorized into different types. Some modules of chemotherapy act by generating oxidative stress or free radicals. They are recognizedvto halt cancer development through cross-linking nucleobases in DNA, consequently harming the DNA, rendering it incapable of uncoiling. The cell is then unable to replicate (7). CPA belongs to this cytotoxic alkylating group of drugs. It is bio-activated by hepatic cytochrome P450 enzymes ensuing in the productionof its two metabolites: phosphoramidemustard and acrolein. Acrolein, a byproductmetabolite of CPA, obstructs the tissue antioxidant defense system, producesreactive oxygen species (ROS), and interacts with protein amino acids causing structural and functional changes (12).

In a study conducted by Kah et.al on adult wistar rats treated with CPA, the liver tissue showed fatty infilteration with normal central vein and portal triad in low doses (3). In this study, venous congestion and mononuclear cell infilteration was seen in periportal area in low doses, but there was no fatty degeneration observed. This difference in studies may be due to the variance in mode of administration which is intra peritoneal injection in the present study whereas oral routeadministration was done in the previous study. In this study, we observed CPA-induced necrotic changes in dose and duration dependent manner. The changes observed were cellular disintegration, distortion and pyknotic nuclei with congestion of blood vessels and leucocyte migrations. The histological comment in the current study was similar to studies conducted by various authors in the past $(3,10,11,12)$. In in-vitro, the endothelial cells lining the sinusoids of the liver are injured by the metabolites of CPA causing veno occlusive disease also known as sinusoidal obstruction syndrome (10). The histological findings in this study such as portal vein engorgement, sinusoidal congestion

DOI: http://dx.doi.org/10.4314/ejhs.v28i6.5 
and subsequently hepatocyte degeneration suggest veno occlusive disease consequent to increase in oxidative stress.

According to researchers, CPA does not show any adverse effect on the kidney. This is because, its by-product, acrolein is not nephrotoxic and is more of urotoxic (14). However, in our study, we have seen pathological changes such as infilteration ofacute inflammatory cells in the cortex, oedema of tubular cells, loss of brush border and finally pyknotic cells. Similar changes were also suggested by Sakr et.al.who injected CPA and observed dilation and congestion of renal blood vessel, vacuolations of epithelial lining renal tubules and atrophy of glomerular tuft. The changes were credited to $\mathrm{CP}$-induced oxidative stress as perceived by the escalation in the oxidative markers in the kidney tissues (15).

The histological changes therefore seen in the liver and kidney tissues in this study may also be attributed to the increase in the oxidative stress of the cell and decrease in the antioxidant agents. Hence, there is the importance of hepatoprotective and renoprotective drug administration, preferably an antioxidant before using chemotherapeutic agents in patients with malignancies and also regular monitoring of liver and kidney function tests in patients undergoing chemotherapeutic regimen.

Our study has demonstrated the effect of aprogressive increase in dosage of CPA in Swiss albino mice and pathological alterations observed in histology of liver and kidney by sequentially increasing both the dosage and duration of treatment.

\section{ACKNOWLEDGEMENTS}

This work was supported by the Department of Anatomy, Kasturba Medical College, Manipal Academy of Higher Education.

\section{REFERENCES}

1. Ahmed A,Hombal S.Cyclophosphamide (Cytoxan). A review on relevant pharmacology and clinical uses. J Am AcadDermatol.1984; 11(6):1115-26.

2. Fraiser L, Kanekal S, Kehrer J. Cyclophosphamide toxicity. Characterising and avoiding the problem.Drugs.1991;42(5):781-95.

3. Khan J, Shahdad S, Makhdoomi M,Hamid S, Bhat M, Jan Y. et.al. Effect of Cyclophoshamide on the microanatomy of liver of albino rats. Int $\mathrm{J}$ Res Med Sci. 2014;2:1466-9.

4. Gaikwad S, Mohan G. Immunomodulatory activity of methanolic extract of thepesiapopulnea leaves in wistar albino rats. Asian Journal of
Pharmaceutical and Clinical Research. 2011;4 (4): 99-101.

5. Wagoner L, Olsen S, Bristow M, O'Connell JB, Taylor DO, Lappe DL et.al. Cyclophoshamide as an alternative to azathioprine in cardiac transplant recipients with suspected azathioprine induced hepatoxicity. Transplantation. 1993; 56(6): 1415-8.

6. Honjo I, Suou T, Hirayama C. Hepatoxicity of cyclophosphamide in man: pharmacokinetic analysis. Res CommunChemPatholPharmacol. 1988; 61(22):149-65.

7. McDonald G, Slattery J,Bouvier M, Ren S, Batchelder AL, Kalhorn TF. Cyclophoshamide metabolism, liver toxicity, and mortality following hematopoietic stem cell transplantation. Blood. 2003;101(5):2043-8.

8. Subramaniam S, CaderR,Mohd R.Low-dose cyclophosphamide-induced acute hepatoxicity. Am J Case Rep. 2013; 14:345.

9. Akay H, Akay T, SecilmisS, Kocak Z, Donderici O. Hepatotoxicity after low dose cyclophosphamide therapy. Al. South Med J. 2006; 99:1399-400.

10. Shokrzadeh M, Ahmadi A, Naghshvar F, Chabra A, JafarinejhadM. Prophylactic Efficacy of Melatonin on Cyclophoshamide-Induced Liver toxicity. Biomed Res Int. 2014.

11. Cuce G, Çetinkaya S, Koc T. Chemoprotective effect of Vitamin E in cyclophosphamide-induced hepatoxicity in rats. ChemBiol Interact. 2015;232:7-11

12. Khorwal G, Chauhan R, Nagar M. Effect of cyclophosphamide on liver in albino rats: a comparative dose dependent histomorphological study. International Journal of Biomedical and Advance Research, 2017;8(3):102-107.

13. D. R. Singh, Principles and Techniques in Histology Microscopy and Photomicrography Published by CBS Publishers and Distributors, $2003,1^{\text {st }}$ edition, pg \# 20-40.

14. Haenen G, Vermeulen N, Tsoi JN, Ragetli HM, Timmerman H, Blast A.Activation of the microsomal glutathione-S-transferase and reduction of the glutathione dependent protection against lipid peroxidation by acrolein. BiochemPharmacol, 1988; 37:1933.

15. Sakr S, El-messady F. Cyclophosphamide Induced Histologial and Immunohistochemical Alterations in Kidney of Albino Rats: The Ameliorative Effect of Fennel Oil. International Journal of Sciences 2017; 6 (08): 78-87. 